\title{
Synthesis, Chemical Reactivity and Fungicidal Activity of Pyrido[1,2-b][1,2,4]triazine Derivatives
}

\author{
Magdy A. Ibrahim, * Reda M. Abdel-Rahman, Ali M. Abdel-Halim, \\ Salah S. Ibrahim and Hassan A. Allimony
}

Department of Chemistry, Faculty of Education, Ain Shams University, Roxy, 11711, Cairo-Egypt

\begin{abstract}
A síntese de alguns novos derivados de pirido[1,2-b][1,2,4]triazinas (2-12) foi obtida através da ciclocondensação de 4-aril-1,6-diamino-2-oxo-1,2-diidropiridina-3,5-dicarbonitrilas (1a,b) com compostos $\alpha, \beta$-bifuncionais. Foram também preparadas pirido[1,2:2',3']triazino[5' $\left.6^{\prime}-\mathrm{f}\right]$ triazinas (13-14). O comportamento de 1a,b frente às interações com indol-2,3-diona e seu análogo $\mathrm{N}$-acetil foi estudado em diferentes condições de reação. As estruturas dos novos produtos foram deduzidas a partir de análise elementar e de dados espectroscópicos (UV, IR, ${ }^{1} \mathrm{H}$ RMN, ${ }^{13} \mathrm{C}$ RMN e espectrometria de massas). Os novos compostos sintetizados foram testados quanto à atividade antifungos.
\end{abstract}

The synthesis of some new pyrido[1,2-b][1,2,4]triazines (2-12) was achieved by cyclocondensation of 4-aryl-1,6-diamino-2-oxo-1,2-dihydropyridine-3,5-dicarbonitriles (1a,b)

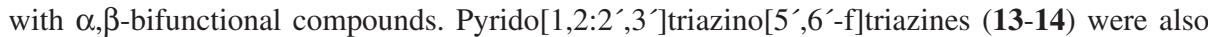
prepared. The behavior of $\mathbf{1 a}, \mathbf{b}$ toward interactions with indole-2,3-dione and its $N$-acetyl analogue have been studied under different reaction conditions. The structures of the new products have been deduced from elemental analysis and spectral data (UV, IR, ${ }^{1} \mathrm{H}$ NMR, ${ }^{13} \mathrm{C}$ NMR and mass spectra). The new synthesized compounds were screened for their antifungal activities.

Keywords: synthesis, $o$-diamines, pyridotriazines, fungicidal activity

\section{Introduction}

Polyfunctional pyridines are highly reactive intermediates that have been extensively used in heterocyclic synthesis. ${ }^{1}$ $o$-Diamines are very active substrates for building of various heterocyclic systems ${ }^{2}$ and are largely used in formation of complexes. ${ }^{3}$ In symmetrical diamines, the product will be the same irrespective of which amine participates first in the reaction. In the case of unsymmetrical diamines, the substituents influence the initial participation of a particular amino group in the reaction, resulting in chemoselective products. The electron withdrawing/ donating nature of substituents in diamine influences the nucleophilicity of the amino group. On the other hand, 1,2,4-triazine derivatives exhibit marked biological and pharmacological effects and are use for the building fused, condensed and isolated heterobicyclic systems. ${ }^{4}$ On the basis of these observations, the objective of this work is the study of the chemical reactivity of 4-aryl-1,6diamino-2-oxo-1,2-dihydropyridine-3,5-dicarbonitriles

*e-mail: magdy_ahmed1977@yahoo.com $(\mathbf{1 a}, \mathbf{b})$ and their use for preparation of nitrogen bridgehead pyrido[1,2-b] $[1,2,4]$ triazines in view of their antifungal activity.

\section{Results and Discussion}

1,6-Diamino-4-(4-chlorophenyl or 3,4,5-trimethoxyphenyl)-2-oxo-1,2-dihydropyridine-3,5-dicarbonitriles (1a,b) have been obtained from refluxing alcoholic solution of 2-cyanoacetohydrazide and arylmethylidinemalononitriles in the presence of few drops of piperidine as a catalyst. ${ }^{5}$ ${ }^{1} \mathrm{H}$ NMR spectra for compounds $\mathbf{1 a}, \mathbf{b}$ showed two signals for each compound at 5.6 and $8.4 \mathrm{ppm}$ characteristic for the $\left(\mathrm{N}-\mathrm{NH}_{2}\right)$ and $\left(\mathrm{C}-\mathrm{NH}_{2}\right)$ protons, respectively. These results indicate the difference in nucleophilicity between the two amino groups. Thus, It is expected the hydrazide $\beta$-nitrogen $\left(\mathrm{N}-\mathrm{NH}_{2}\right)$ more nucleophilic and would react more rapidly with the electron deficient carbon than the amino group at carbon atom $\left(\mathrm{C}-\mathrm{NH}_{2}\right)$. Mass spectra for compounds $\mathbf{1 a}$ and $\mathbf{1 b}$ showed the molecular ion peaks at $\mathrm{m} / \mathrm{z} 341$ and 285, respectively (the base peaks), indicating the high stability the pyridine moiety. Heterocyclic systems 
containing 1,2-diamine centers ${ }^{6}$ are used for building fused heterocyclic systems via a nitrogen bridge. Thus, the regio-isomeric 8-aryl-2,6-dioxo-1,3,4,6-tetrahydro2H-pyrido[1,2-b][1,2,4]triazine-7,9-dicarbonitrile (2) and 3,6-dioxo-pyrido[1,2-b][1,2,4]triazine-7,9-dicarbonitrile (3) have been obtained from alkylation and acylation of compound $\mathbf{1 b}$ with monochloroacetic acid and chloroacetyl chloride, respectively (Scheme 1). ${ }^{1} \mathrm{H}$ NMR spectra of compounds $\mathbf{2}$ and $\mathbf{3}$ showed signals for each $\mathrm{CH}_{2}$ protons at 2.91 and $3.23 \mathrm{ppm}$, respectively, while their ${ }^{13} \mathrm{C}$ NMR spectra exhibited signals for each $\mathrm{CH}_{2}$ carbons at 36.66 and 40.05 ppm, respectively.

Heterocyclization of diaminopyridones $\mathbf{1 a}, \mathbf{b}$ with phenacyl bromide ${ }^{7}$ in refluxing aqueous $\mathrm{NaOH}$ yielded 8-aryl-1,2,5,6-tetrahydro-6-oxo-3-phenyl-pyrido[1,2-b] [1,2,4]triazine-7,9-dicarbonitriles $(\mathbf{4 a}, \mathbf{b})$, while the perhydropyridotriazine $\mathbf{5}$ was obtained from treatment of compound $\mathbf{1 b}$ with 1,2-dibromoethane in alcoholic<smiles>N#Cc1c(-c2ccc(Cl)cc2)c(C#N)c(=O)n2c1NCC(=O)N2</smiles>

3

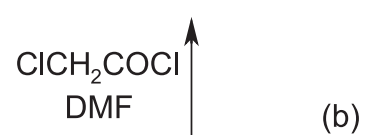<smiles>N#Cc1c(-c2ccc(Cl)cc2)c(C#N)c(=O)n2c1NC(=O)CN2</smiles>

2

\section{$\uparrow$}

$\mathrm{ClCH}_{2} \mathrm{COOH}$

(b) DMF<smiles>N#Cc1c(Br)c(C#N)c(=O)n(N)c1N</smiles><smiles>N#Cc1c([Al])c(C#N)c(=O)n2c1NCC(c1ccccc1)=N2</smiles>

$1 a, b$

$4 a, b$<smiles></smiles>

1a, $\mathrm{Ar}=$<smiles>COc1cc(I)cc(OC)c1OC</smiles>

1b, $A r=$<smiles>Cc1ccc(Cl)cc1</smiles>

Scheme 1 
$\mathrm{KOH}$ (Scheme 1). The IR spectra of $\mathbf{4 a}$ and $\mathbf{4 b}$ showed absorption bands at 3445 and $3320 \mathrm{~cm}^{-1}$ assigned to $\mathrm{NH}$ groups, respectively, while the IR spectrum of $\mathbf{5}$ showed two absorption bands at 3327 and $3243 \mathrm{~cm}^{-1}$ for two $\mathrm{NH}$ groups.

Some new 8-aryl-2,6-dioxo-1,2-dihydropyrido[1,2-b] $[1,2,4]$ triazine-7,9-dicarbonitriles (6a-g) have been synthesized by cyclocondensation of compounds $\mathbf{1 a}, \mathbf{b}$ with $\alpha$-oxocarboxylic acids, namely pyruvic, $\alpha$-oxobutyric, 4-chlorostyrylglyoxalic and phenoxypyruvic acids in refluxing glacial acetic acid (Scheme 2). It should be noted that this reaction occurred preferentially between the $\mathrm{N}^{1}$-amino group $\left(\mathrm{N}-\mathrm{NH}_{2}\right)$ and the $\alpha$-keto function of the electrophile to form a hydrazone intermediate, which underwent a cyclodehydration reaction between the other amino group at $\mathrm{C}^{6}\left(\mathrm{C}-\mathrm{NH}_{2}\right)$ and the hydroxyl group of the acid function affording the target pyridotriazine derivatives 6a-g. ${ }^{13} \mathrm{C}$ NMR spectra gave good evidence for the formation of compounds 6a-g. For examples, the ${ }^{13} \mathrm{C}$ NMR spectrum of compound $\mathbf{6 a}$ showed a new signal at $18.60 \mathrm{ppm}$ characteristic for a methyl group in position 3 . In the case of compound $\mathbf{6} \mathbf{f}$ the vinyl carbons were observed in the spectrum in their expected positions at 122.75 and $135.70 \mathrm{ppm}$ for $\mathrm{C}_{\alpha}$ and $\mathrm{C}_{\beta}$, respectively.

Cyclic 1,2-bioxygen compounds were also used for building various fused heterocyclic systems. ${ }^{8}$ Thus, compounds $\mathbf{7 a}, \mathbf{b}$ were prepared from refluxing compound 1a,b with diethyl oxalate in dry dioxane and/or with oxalyl chloride in warm DMF (Scheme 2).

Some new pyridotriazines were obtained from cyclocondensation of 1,6-diaminopyridones $\mathbf{1 a}, \mathbf{b}$ with $\alpha$-dicarbonyl compounds. Thus, treatment of $\mathbf{1 a}$ with butane-2,3-dione in glacial acetic acid afforded 2,3-dimethylpyrido[1,2-b][1,2,4]triazine (8), while the corresponding 2,3-diphenylpyridotriazine derivatives $\mathbf{9 a , b}$ were obtained from refluxing 1a,b with benzil in glacial acetic acid. Dihydro analogues 10a,b were obtained by refluxing compounds $\mathbf{1 a}, \mathbf{b}$ with benzoin under the same reaction conditions. Oxidation of compounds $\mathbf{1 0 a}, \mathbf{b}$ in methanolic ferric chloride ${ }^{9}$ produced compounds $9 \mathbf{a}, \mathbf{b}$ (the same mp and mixed mp) (Scheme 2). ${ }^{1} \mathrm{H}$ NMR of compound $10 \mathrm{~b}$ showed a signal at $5.62 \mathrm{ppm}$ assigned to $\mathrm{CH}$ proton in position 2, while its ${ }^{13} \mathrm{C}$ NMR spectrum showed a signal at $77.83 \mathrm{ppm}$ characteristic for the corresponding carbon atom.

Chlorination of compound $7 \mathbf{a}$ using phosphorus oxychloride afforded 2,3-dichloro-6-oxo-8- (3,4,5trimethoxy)-6H-pyrido[1,2-b][1,2,4]triazine-7,9dicarbonitrile (11) (Scheme 3).

Compounds $\mathbf{7 a}, \mathbf{b}$ were used as starting materials for the synthesis of fused heteropolycyclic systems. Thus, hydroxymethylation of $\mathbf{7 a , b}$ by refluxing with methanolformaldehyde solution produced 8-aryl-2,3,6-trioxo-1,4dihydroxymethyl-1,2,3,4,5,6-hexa- hydropyrido[1,2-b] [1,2,4]triazine-7,9-dicarbonitriles (12a,b), which upon heterocyclization by refluxing with thiosemicarbazide in acetic acid led to 7-oxo-2-thioxo-2,3,5,6,7,11-

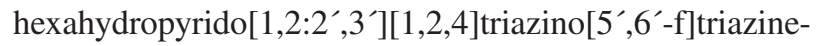
8,10-dicarbonitriles(13a, b). Hydrazinolysis of 13b in boiling ethanol furnished the hydrazinotriazine $\mathbf{1 4}$ (Scheme 3).

The course of the reactions of cyclic 1,2-bi-oxygen heterocyclic compounds with aromatic heterocyclic $o$-diamines was shown to depend on the reaction conditions, type of solvent and also the substituents in the diamino compounds. ${ }^{10}$ Thus, reaction of compounds 1a,b with indole-2,3-dione (isatine) in different media can yield different products. Treating 1a with indole-2,3dione in absolute ethanol and few drops of piperidine produced the Schiff base condensate, 6-amino-4-(3,4,5trimethoxyphenyl)-2-oxo-1-[2-oxo-1,2-dihydro-3-indolo3-ylidine)amino]-1,2-dihydropyridine-3,5-dicarbonitrile (15). Alternatively, warming indole-2,3-dione with alcoholic $\mathrm{NaOH}$ solution yielded a 2-aminophenylglyoxalic acid that adds to 1,6-diaminopyridinone $\mathbf{1 b}$ to give the condensation product 16. Indolotriazinopyridines 17a,b were produced from ring closure reaction of compounds 15 and/or 16 in boiling glacial acetic acid in the presence of freshly fused sodium acetate. Acetylation of compound 17a by refluxing with acetic anhydride afforded the $N$-acetyl derivative 18 (Scheme 4). IR spectrum of 18 indicated that $\mathrm{NH}$ group disappeared and a new characteristic band at $1734 \mathrm{~cm}^{-1}$ appeared for the $\mathrm{C}=\mathrm{O}$ of the acetyl group. The mass spectrum revealed the parent peak at $\mathrm{m} / \mathrm{z} 494$ which is coincident with the formula weight in agreement with the postulated structure.

$\mathrm{N}$-acetylisatine showed a different behavior. ${ }^{11}$ Reaction of $1 \mathbf{a}$ with $N$-acetylisatine in absolute ethanol in the presence of few drops of piperidine led to 8-(3,4,5-trimethoxyphenyl)2-(2-acetanilido)-3,6-dioxo-3,6-dihydro-4H-pyrido[1,2-b] $[1,2,4]$ triazine-7,9-dicarbonitrile $(\mathbf{2 0})$ and not to the isomeric product 21 (Scheme 4). This reaction can be explained by an increase in the positive charge on the $\alpha$-carbon atom in comparison to isatine itself due to the electron withdrawing acetyl group which facilitates the nucleophilic attack of more nucleophilic amino group $\left(\mathrm{N}-\mathrm{NH}_{2}\right)$ at this position with concomitant opening of five membered ring. Apparently, the reaction can be claimed to proceed via intermediate $\mathbf{1 9}$, as also observed by previous workers in reaction with other diamines. ${ }^{12}$ However, this type of intermediate was reported to be unstable and not isolated. 
<smiles>COc1cc(-c2c(C#N)c(=O)n3nc(C)c(C)nc3c2C#N)cc(OC)c1OC</smiles><smiles>N#Cc1c(Br)c(C#N)c(=O)n2c1NC(c1ccccc1)C(c1ccccc1)=N2</smiles><smiles>CC(=O)C(C)=O</smiles>

(a)

9a,b

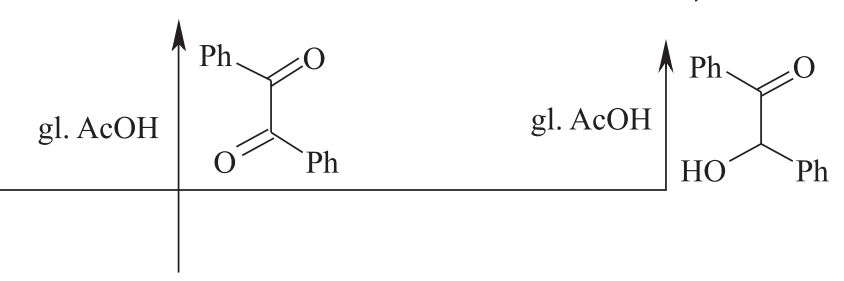<smiles></smiles>

1a,b<smiles></smiles>

7a,b<smiles>COc1cc(C#[W][Ba])cc(OC)c1OC</smiles><smiles>[R]C(=O)C(=O)O</smiles><smiles>[R]c1nn2c(=O)c(C#N)c([Al])c(C#N)c2[nH]c1=O</smiles>

6a-g

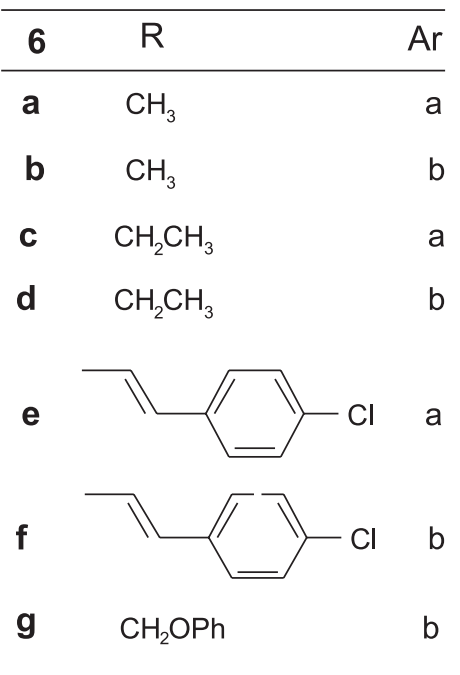


<smiles>N#Cc1c(Br)c(C#N)c2[nH]c(=O)c(=O)[nH]n2c1=O</smiles>

7a,b<smiles>COc1cc(-c2c(C#N)c(=O)n3nc(Cl)c(Cl)nc3c2C#N)cc(OC)c1OC</smiles><smiles></smiles>

$12 a, b$<smiles>[Y]C(=O)CC1NCCC1N</smiles><smiles>N#Cc1c(Br)c(C#N)c(=O)n2c1N(CO)c1nc(=S)[nH]nc1N2CO</smiles>

$13 a, b$

(b)<smiles>NCCOCNN</smiles><smiles>N#Cc1c(-c2ccc(Cl)cc2)c(C#N)c(=O)n2c1N(CO)c1nc(NN)nnc1N2CO</smiles>

14

$\mathrm{b}, \mathrm{Ar}=$

$\mathrm{OMe}$

\section{Fungicidal activity}

Several new synthesized compounds were screened for their antifungal activities against two species of fungi, namely Alternaria alternata and Aspergillus niger using the disc diffusion method. ${ }^{13}$ The tested compounds were dissolved in DMF (which has no inhibitory activity) to get concentrations of $1 \mathrm{mg} \mathrm{mL}^{-1}$ solution. The antibiotic fluconazole was used as standard antifungal reference. The inhibition zones of microbial growth surrounding the filter paper disc $(2.5 \mathrm{~mm})$ were measured in millimeters at the end of an incubation period at $30 \mathrm{C}$ for 3 days. Inhibition of the organisms was evidenced by a clear zone surrounding each disk (Table 1).

All the tested compounds showed variable activities toward the two species of fungi, some of them comparable to standard fluconazole. The most active triazines were $\mathbf{2 d}$, $3,9 \mathrm{~b}$ and $\mathbf{1 0 .}$.

From the results obtained, it is clear that increasing the percentage of nitrogen in the tested compounds led to
Table 1. Fungicidal activity of some of the new compounds 1-20

\begin{tabular}{lccc}
\hline Compound & $\begin{array}{c}\text { Nitrogen content } \\
\text { N / \% }\end{array}$ & $\begin{array}{c}\text { Diameter of inhibition zone } \\
\text { Alternaria } \\
\text { alternata }\end{array}$ & $\begin{array}{c}\text { Aspergillus } \\
\text { niger }\end{array}$ \\
\hline 1a & 20.5 & + & ++ \\
2d & 16.5 & ++ & +++ \\
$\mathbf{3}$ & 21.6 & +++ & +++ \\
$\mathbf{4}$ & 21.6 & ++ & ++ \\
$\mathbf{5 b}$ & 18.5 & ++ & ++ \\
$\mathbf{6 b}$ & 20.5 & ++ & ++ \\
$\mathbf{8 b}$ & 17.5 & ++ & ++ \\
$\mathbf{9 b}$ & 24.6 & ++ & +++ \\
$\mathbf{1 0}$ & 30.9 & +++ & +++ \\
$\mathbf{1 4}$ & 22.4 & ++ & ++ \\
$\mathbf{1 7}$ & 18.5 & + & + \\
(fluconazole) & & +++ & +++ \\
\hline
\end{tabular}

Lower active $=+($ inhibition zone $1-10 \mathrm{~mm})$, moderately active $=$ (inhibition zone $11-25 \mathrm{~mm}$ ) and highly active $=+++$ (inhibition zone $>$ $25 \mathrm{~mm})$. 
<smiles>COc1cc(-c2c(C#N)c(=O)n3nc4c5ccc(C(C)(C)C)cc5n(OC)c4nc3c2C#N)cc(OC)c1OC</smiles>

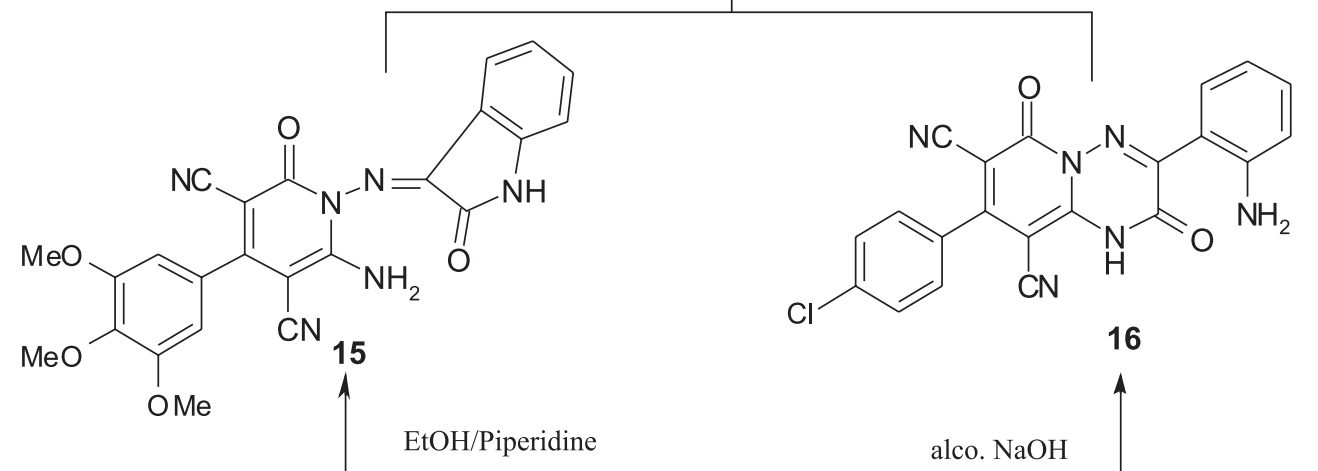

(a)

(b)<smiles>O=C1Nc2ccccc2C1=O</smiles>

$1 a, b$

(a)<smiles></smiles><smiles>CC(=O)N1C(=O)C(=O)c2ccccc21</smiles><smiles>COc1cc(-c2cn(NC(=O)C(=O)c3ccccc3NC(C)=O)c(=O)c(N)c2C#N)cc(OC)c1OC</smiles><smiles></smiles>

Scheme 4

higher effects toward the tested fungi. The antifungal effects decrease in the order of: $\mathbf{1 0}>\mathbf{9 b}>\mathbf{3}>\mathbf{2 d}$ for higher activity and $\mathbf{1 4}>\mathbf{6 b}>\mathbf{4}>\mathbf{8 b}>\mathbf{5 b}>\mathbf{1 a}$ for moderate activity. The lower activity was observed by compound $\mathbf{1 7 a}$ (Table 1). 


\section{Conclusion}

Cyclocondensation of 4-aryl-1,6-diamino-2-oxo-1,2dihydropyridine-3,5-dicarbonitriles with $\alpha, \beta$-bifunctional compounds takes place regioselectively through condensation of $\left(\mathrm{N}-\mathrm{NH}_{2}\right)$ group with the more electrophilic carbon center followed by cyclization to produce several new pyrido[1,2-b][1,2,4]triazine derivatives.

\section{Experimental}

Melting points are uncorrected and were recorded in open capillary tubes on a Stuart SMP3 melting point apparatus. Infrared spectra were recorded on FT-IR Bruker Vector 22 spectrophotometer using $\mathrm{KBr}$ wafer technique. UV absorption spectra (DMF) were recorded on a Jasco model (V-550) UV spectrophotometer. ${ }^{1} \mathrm{H}$ NMR and ${ }^{13} \mathrm{C}$ NMR spectra were measured on Gemini $(200 \mathrm{MHz})$ spectrometer and Bruker $(250 \mathrm{MHz}) \mathrm{AC}$ spectrometer using DMSO- $d_{6}$ as solvent and TMS (chemical shift in $\mathrm{ppm}$ ) as an internal standard. Mass spectra were obtained using a Shimadzu GCMS qp 1000 ex instrument mass spectrometer $(70 \mathrm{eV})$.

4-Aryl-1,6-diamino-2-oxo-1,2-dihydropyridine-3,5dicarbonitriles $(\mathbf{1} \boldsymbol{a}, \boldsymbol{b})$

Compounds $\mathbf{1 a}$ and $\mathbf{1 b}$ have been prepared according to the reported method. ${ }^{5}$

\section{Compound $1 \mathrm{a}$}

Crystallized from DMF as white crystals, yield 80\%, mp 255-256 ${ }^{\circ} \mathrm{C}$. UV $\lambda_{\max } / \mathrm{nm}(\log \varepsilon): 344$ (3.39), 276 (3.58). IR (KBr) $v_{\max } / \mathrm{cm}^{-1}: 3334,3194\left(2 \mathrm{NH}_{2}\right), 2998$, 2941, $2839\left(\mathrm{CH}_{3}\right.$ groups $), 2215(2 \mathrm{C} \equiv \mathrm{N}), 1669(\mathrm{C}=\mathrm{O})$, $1633\left(\right.$ def. $\left.\mathrm{NH}_{2}\right), 1591(\mathrm{C}=\mathrm{N}), 1513(\mathrm{C}=\mathrm{C}), 1466,1416$ (def. $\left.\mathrm{CH}_{3}\right)$. ${ }^{1} \mathrm{H}$ NMR $\left(\delta\right.$, DMSO): 3.78 (s, $\left.3 \mathrm{H}, \mathrm{CH}_{3} \mathrm{O}\right), 3.81$ (s, $\left.6 \mathrm{H}, 2 \mathrm{CH}_{3} \mathrm{O}\right), 5.60$ (s, 2H, N-NH $)_{2}, 6.82(\mathrm{~s}, 2 \mathrm{H}, \mathrm{Ar}-\mathrm{H})$, $8.40 \mathrm{ppm}\left(\mathrm{s}, 2 \mathrm{H}, \mathrm{C}-\mathrm{NH}_{2}\right) .{ }^{13} \mathrm{C}$ NMR ( $\delta$, DMSO): 56.47 $\left(2 \mathrm{CH}_{3} \mathrm{O}\right), 60.47\left(\mathrm{CH}_{3} \mathrm{O}\right), 74.63\left(\mathrm{C}_{5}-\mathrm{CN}\right), 86.67\left(\mathrm{C}_{3}-\mathrm{CN}\right)$, $116.01(\mathrm{C} \equiv \mathrm{N}), 116.88(\mathrm{C} \equiv \mathrm{N}), 106.32\left(\mathrm{C} 2{ }^{\prime}\right.$ and $\left.\mathrm{C} 6{ }^{\circ}\right), 129.94$ (C1'), 139.03 (C4`), 153.00 (C3` and C5'), $156.98\left(\mathrm{C}_{4}\right)$, $159.62\left(\mathrm{C}_{6}\right), 162.65 \mathrm{ppm}\left(\mathrm{C}_{2}\right.$ as $\left.\mathrm{C}=\mathrm{O}\right) . \mathrm{M} / \mathrm{z}(\mathrm{Int} . \%) 342$ (20.1), 341 (100), 326 (49.18), 298 (20.77), 283 (8.89), 268 (10.06), 236 (2.82), 168 (2.88). Anal. Calc. for $\mathrm{C}_{16} \mathrm{H}_{15} \mathrm{~N}_{5} \mathrm{O}_{4}$ (341.3): C, 59.96; H, 2.39; N, 15.21. Found: C, 59.34; H, $2.62 ; \mathrm{N}, 15.34$.

\section{Compound $\mathbf{1 b}$}

Crystallized from dioxane as white crystals, yield 90\%, $\mathrm{mp}>300^{\circ} \mathrm{C}$. IR (KBr) $v_{\text {max }} / \mathrm{cm}^{-1}: 3455,3400,3350,3310$
(2 $\left.\mathrm{NH}_{2}\right), 2222(2 \mathrm{C} \equiv \mathrm{N}), 1665(\mathrm{C}=\mathrm{O}), 1624\left(\right.$ def. $\left.\mathrm{NH}_{2}\right)$, $1590(\mathrm{C}=\mathrm{N}), 1562(\mathrm{C}=\mathrm{C}) .{ }^{1} \mathrm{H}$ NMR $(\delta, \mathrm{DMSO}): 5.60$ (s, $2 \mathrm{H}, \mathrm{N}_{-} \mathrm{NH}_{2}$ ), 7.63 (d, 2H, Ar-H), 7.84 (d, 2H, Ar-H), 8.40 ppm (s, 2H, C-NH $\mathrm{N}_{2}$ ). Anal. Calc. for $\mathrm{C}_{13} \mathrm{H}_{8} \mathrm{ClN}_{5} \mathrm{O}(285.69)$ : C, 54.60; H, 2.80; N, 24.51. Found: C, 54.59; H, 2.86; N, 24.53 .

8-(4-Chlorophenyl)-2,6-dioxo-1,3,4,6-tetraahydro- $2 \mathrm{H}$ pyrido[1,2-b][1,2,4]triazine-7,9-dicarbonitrile (2)

A mixture of $\mathbf{1 b}(10 \mathrm{mmol})$ and monochloroacetic acid $(10 \mathrm{mmol})$ in DMF $(50 \mathrm{~mL})$ was refluxed for $4 \mathrm{~h}$, after cooling the reaction mixture was poured onto ice. The solid obtained was filtered and crystallized from methanol to give 2 as yellow crystals, yield $66 \%, \mathrm{mp}>300^{\circ} \mathrm{C}$. IR $(\mathrm{KBr}) v_{\max }$ ' $\mathrm{cm}^{-1}$ : $3397(\mathrm{OH}), 3265(\mathrm{NH}), 2218(2 \mathrm{C} \equiv \mathrm{N}), 1640(\mathrm{C}=\mathrm{O})$, $1522(\mathrm{C}=\mathrm{C}), 1491,1465\left(\right.$ def. $\left.\mathrm{CH}_{2}\right) .{ }^{1} \mathrm{H}$ NMR $(\delta$, DMSO): $2.91\left(\mathrm{~s}, 2 \mathrm{H}, \mathrm{CH}_{2}\right), 5.75(\mathrm{~s}, 1 \mathrm{H}, \mathrm{NH}), 7.52(\mathrm{~d}, 2 \mathrm{H}, \mathrm{Ar}-\mathrm{H}), 7.73$ (d, $2 \mathrm{H}, \mathrm{Ar}-\mathrm{H}), 8.51 \mathrm{ppm}$ (s, $1 \mathrm{H}, \mathrm{OH}$ of 1,2,4-triazin-5-ol). ${ }^{13} \mathrm{C}$ NMR ( $\delta$, DMSO): $36.66\left(\mathrm{CH}_{2}\right), 75.19\left(\mathrm{C}_{9}-\mathrm{CN}\right), 87.29$ $\left(\mathrm{C}_{7}-\mathrm{CN}\right), 116.22(\mathrm{C} \equiv \mathrm{N}), 117.09(\mathrm{C} \equiv \mathrm{N}), 129.65,130.89$, 134.31 and 135.95 (6C of aryl carbons), $157.48\left(\mathrm{C}_{8}\right)$, $159.27\left(\mathrm{C}_{9 \mathrm{a}}\right), 159.99\left(\mathrm{C}_{2}\right.$ as $\left.\mathrm{C}=\mathrm{O}\right), 161 \mathrm{ppm}\left(\mathrm{C}_{6}\right.$ as $\left.\mathrm{C}=\mathrm{O}\right)$. Anal. Calc. for $\mathrm{C}_{15} \mathrm{H}_{8} \mathrm{ClN}_{5} \mathrm{O}_{2}$ (325.72): $\mathrm{C}, 55.13 ; \mathrm{H}, 2.48$; $\mathrm{N}, 21.50$. Found: C, 55.00; H, 2.72; N, 21.81.

8-(4-Chlorophenyl)-3,6-dioxo-1,3,4,6-hexahydro- $\mathrm{H}$ pyrido[1,2-b][1,2,4]triazine-7,9-dicarbonitrile (3)

Compound 1b (10 mmol) was dissolved in DMF $(50 \mathrm{~mL})$, chloroacetyl chloride $(10 \mathrm{mmol})$ was added dropwise within $15 \mathrm{~min}$, then refluxed for $4 \mathrm{~h}$. After cooling the reaction mixture was poured onto ice. The solid obtained was filtered and crystallized from ethanol to give 3 as yellow crystals, yield $52 \%, \mathrm{mp} 235-236^{\circ} \mathrm{C}$. IR ( KBr) $v_{\max } / \mathrm{cm}^{-1}: 3450(\mathrm{br}, \mathrm{OH} \rightleftharpoons \mathrm{NH}), 2967\left(\mathrm{CH}_{2}\right), 2214$ (2 $\mathrm{C} \equiv \mathrm{N}), 1650(\mathrm{C}=\mathrm{O}), 1560(\mathrm{C}=\mathrm{C}), 1492,1423\left(\right.$ def. $\left.\mathrm{CH}_{2}\right)$. ${ }^{1} \mathrm{H}$ NMR ( $\delta$, DMSO): 3.23 (s, 2H, $\mathrm{CH}_{2}$ ), 5.30, 5.40 (each s, $2 \mathrm{H}, 2 \mathrm{NH}), 8.23$ (d, $2 \mathrm{H}, \mathrm{Ar}-\mathrm{H}), 8.42 \mathrm{ppm}(\mathrm{d}, 2 \mathrm{H}, \mathrm{Ar}-\mathrm{H}) .{ }^{13} \mathrm{C}$ NMR ( $\delta$, DMSO): $40.05\left(\mathrm{CH}_{2}\right), 83.01\left(\mathrm{C}_{9}-\mathrm{CN}\right), 89.19\left(\mathrm{C}_{7}-\right.$ $\mathrm{CN}), 122.13(\mathrm{C} \equiv \mathrm{N}), 123.46(\mathrm{C} \equiv \mathrm{N}), 134.20,135.63,136.11$ and 140.05 (6C of aryl carbons), $140.20\left(\mathrm{C}_{8}\right), 159.87\left(\mathrm{C}_{9 \mathrm{a}}\right)$, $161.50\left(\mathrm{C}_{3}\right.$ as $\left.\mathrm{C}=\mathrm{O}\right), 162.39 \mathrm{ppm}\left(\mathrm{C}_{6}\right.$ as $\left.\mathrm{C}=\mathrm{O}\right)$. Anal. Calc. for $\mathrm{C}_{15} \mathrm{H}_{8} \mathrm{ClN}_{5} \mathrm{O}_{2}$ (325.72): C, 55.13; H, 2.48; N, 21.50. Found: C, 54.99; H, 2.72; N, 21.81.

8-Aryl-1,2,5,6-tetrahydro-6-oxo-3-phenyl-pyrido[1,2-b] [1,2,4]triazine-7,9-dicarbonitriles $(\mathbf{4 a , b})$

A mixture of $\mathbf{1 a}$ or $\mathbf{1 b}$ ( $5 \mathrm{mmol})$ and phenacyl bromide $(5 \mathrm{mmol})$ was refluxed in aqueous $\mathrm{NaOH}(5 \%, 50 \mathrm{~mL})$ for 
$4 \mathrm{~h}$, after cooling the reaction mixture was neutralized with conc. $\mathrm{HCl}$. The solid obtained was filtered, washed several times with water and crystallized to give $\mathbf{4 a , b}$.

\section{Compound $4 a$}

Crystallized from ethanol as white crystals, yield 74\%, mp 195-196 ${ }^{\circ} \mathrm{C}$. IR (KBr) $v_{\max } / \mathrm{cm}^{-1}: 3445(\mathrm{NH}), 2940,2838$ $\left(\mathrm{CH}_{3}\right.$ and $\left.\mathrm{CH}_{2}\right), 2211(2 \mathrm{C} \equiv \mathrm{N}), 1653(\mathrm{C}=\mathrm{O}), 1588(\mathrm{C}=\mathrm{N})$, $1508(\mathrm{C}=\mathrm{C}), 1459,1417\left(\right.$ def. $\left.\mathrm{CH}_{2}\right) .{ }^{1} \mathrm{H}$ NMR $(\delta, \mathrm{DMSO})$ : $3.31\left(\mathrm{~s}, 2 \mathrm{H}, \mathrm{CH}_{2}\right), 4.43$ (s, $\left.3 \mathrm{H}, \mathrm{CH}_{3} \mathrm{O}\right), 4.52\left(\mathrm{~s}, 3 \mathrm{H}, \mathrm{CH}_{3} \mathrm{O}\right)$, $4.64\left(\mathrm{~s}, 3 \mathrm{H}, \mathrm{CH}_{3} \mathrm{O}\right), 6.22(\mathrm{~s}, 1 \mathrm{H}, \mathrm{NH}$ exchangeable with $\left.\mathrm{D}_{2} \mathrm{O}\right), 7.59$ (s, $\left.2 \mathrm{H}, \mathrm{Ar}-\mathrm{H}\right), 8.36 \mathrm{ppm}(\mathrm{s}, 5 \mathrm{H}, \mathrm{Ar}-\mathrm{H})$. Anal. Calc. for $\mathrm{C}_{24} \mathrm{H}_{19} \mathrm{~N}_{5} \mathrm{O}_{4}$ (441.45): C, 65.30; H, 4.34; N, 15.86 . Found: C, 65.13; H, 4.25; N, 15.69 .

\section{Compound $\mathbf{4 b}$}

Crystallized from ethanol as white crystals, yield 71\%, mp 143-144 ${ }^{\circ} \mathrm{C}$. IR $(\mathrm{KBr}) v_{\max } / \mathrm{cm}^{-1}: 3320(\mathrm{NH}), 2980\left(\mathrm{CH}_{2}\right)$, $2208(\mathrm{C} \equiv \mathrm{N}), 1636(\mathrm{C}=\mathrm{O}), 1591(\mathrm{C}=\mathrm{N}), 1516(\mathrm{C}=\mathrm{C}), 1495$, 1443 (def. $\mathrm{CH}_{2}$ ). Anal. Calc. for $\mathrm{C}_{21} \mathrm{H}_{12} \mathrm{ClN}_{5} \mathrm{O}$ (385.81): C, 65.32; H, 3.11; N, 18.14. Found: C, 64.89; H, 3.07; N, 18.12 .

8-(4-Chlorophenyl)-6-oxo-1,3,4,6-tetrahydro-2Hpyrido[1,2-b][1,2,4]triazine-7,9-dicarbonitrile (5)

A mixture of $\mathbf{1 b}(5 \mathrm{mmol})$ and 1,2-dibromoethane $(5 \mathrm{mmol})$ in alcoholic $\mathrm{KOH}(5 \%, 50 \mathrm{~mL})$ was refluxed for $4 \mathrm{~h}$, cooled and neutralized with conc. HCl. The solid so formed was filtered, washed with water and crystallized from methanol to give $\mathbf{5}$ as white crystals, yield $67 \%$, $\mathrm{mp}>300{ }^{\circ} \mathrm{C}$. IR (KBr) $v_{\max } / \mathrm{cm}^{-1}: 3327$ and $3243(2 \mathrm{NH})$, $2900\left(\mathrm{CH}_{2}\right), 2210(2 \mathrm{C} \equiv \mathrm{N}), 1649(\mathrm{C}=\mathrm{O}), 1581(\mathrm{C}=\mathrm{N}) .{ }^{1} \mathrm{H}$ NMR ( $\delta$, DMSO): 3.31 (m, 4H, $2 \mathrm{CH}_{2}$ ), 4.98-5.06 (bs, $2 \mathrm{H}$, 2NH exchangeable with $\left.\mathrm{D}_{2} \mathrm{O}\right), 8.22(\mathrm{~d}, 2 \mathrm{H}, \mathrm{Ar}-\mathrm{H}), 8.40$ ppm (d, 2H, Ar-H). Anal. Calc. for $\mathrm{C}_{15} \mathrm{H}_{10} \mathrm{ClN}_{5} \mathrm{O}$ (311.73): C, 57.80; H, 3.23; N, 22.47. Found: C, 57.68; H, 3.17; N, 22.32 .

8-Aryl-2,6-dioxo-3-substituted-1,2,5,6-tetrahydropyrido [1,2-b][1,2,4]triazine-7,9-dicarbonitriles (6a-g)

A mixture of $\mathbf{1 a}$ or $\mathbf{1 b}(10 \mathrm{mmol})$ and acyclic 1,2-bioxo compounds such as pyruvic acid, $\alpha$-oxobutyric acid, $p$-chlorostyryl glyoxalic acid and phenoxy pyruvic acids $(10 \mathrm{mmol})$ in glacial acetic acid $(30 \mathrm{~mL})$ was refluxed for $4 \mathrm{~h}$. The solid obtained after cooling was filtered, washed with water and crystallized from a proper solvents to give 6a-g. IR $(\mathrm{KBr}) \mathrm{v}_{\max } / \mathrm{cm}^{-1}$ of 6a-g: $3463(\mathrm{OH} \rightleftharpoons \mathrm{NH}), 2211-2219(2 \mathrm{C} \equiv \mathrm{N}), 1695-1633$ (2 $\mathrm{C}=\mathrm{O})$.

\section{Compound $\mathbf{6 a}$}

Crystallized from ethanol as white crystals, yield 90\%, $\mathrm{mp}>300^{\circ} \mathrm{C} .{ }^{1} \mathrm{H}$ NMR $\left(\delta\right.$, DMSO): $1.60\left(\mathrm{~s}, 3 \mathrm{H}, \mathrm{CH}_{3}\right), 2.31$ (s, $1 \mathrm{H}, \mathrm{NH}$ of triazinone), $3.76\left(\mathrm{~s}, 3 \mathrm{H}, \mathrm{CH}_{3} \mathrm{O}\right), 3.82(\mathrm{~s}, 6 \mathrm{H}$, $\left.2 \mathrm{CH}_{3} \mathrm{O}\right), 6.81 \mathrm{ppm}(\mathrm{s}, 2 \mathrm{H}, \mathrm{Ar}-\mathrm{H}) .{ }^{13} \mathrm{C}$ NMR $(\delta$, DMSO): $18.60\left(\mathrm{CH}_{3}\right), 56.99\left(2 \mathrm{CH}_{3} \mathrm{O}\right), 60.99\left(\mathrm{CH}_{3} \mathrm{O}\right), 82.00\left(\mathrm{C}_{9}-\right.$ $\mathrm{CN}), 86.10\left(\mathrm{C}_{7}-\mathrm{CN}\right), 107.04\left(\mathrm{C}^{`}{ }^{`}\right.$ and $\left.\mathrm{C}^{\circ}\right), 118.12(2$ $\mathrm{C} \equiv \mathrm{N}), 132.24\left(\mathrm{Cl}^{\circ}\right), 137.00\left(\mathrm{C}^{`}\right), 153.41$ (C3`and $\left.\mathrm{C} 5^{`}\right)$, $154.09(\mathrm{C} 3), 155.02\left(\mathrm{C}_{8}\right), 157.16\left(\mathrm{C}_{9 \mathrm{a}}\right), 159.00\left(\mathrm{C}_{2}\right.$ as $\mathrm{C}=\mathrm{O}), 161.05 \mathrm{ppm}\left(\mathrm{C}_{6}\right.$ as $\left.\mathrm{C}=\mathrm{O}\right)$. Anal. Calc. for $\mathrm{C}_{19} \mathrm{H}_{15} \mathrm{~N}_{5} \mathrm{O}_{5}$ (393.36): C, 58.02; H, 3.84; N, 17.80. Found: C, 57.98; H, $3.77 ; \mathrm{N}, 17.74$.

\section{Compound $\boldsymbol{6} \boldsymbol{b}$}

Crystallized from ethanol as white crystals, yield $88 \%$, $\mathrm{mp}>300^{\circ} \mathrm{C} .{ }^{1} \mathrm{H}$ NMR $\left(\delta\right.$, DMSO): 1.91 (s, $\left.3 \mathrm{H}, \mathrm{CH}_{3}\right), 3.43$ (bs, 1H, NH of triazinone), 7.53 (d, 2H, Ar-H), $7.68 \mathrm{ppm}$ (d, 2H, Ar-H).

\section{Compound $\mathbf{6 c}$}

Crystallized from methanol as white crystals, yield 91\%, mp 130-131 ${ }^{\circ} \mathrm{C}$. Anal. Calc. for $\mathrm{C}_{20} \mathrm{H}_{17} \mathrm{~N}_{5} \mathrm{O}_{3}$ (407.39): C, 58.97; H, 4.21; N, 17.19. Found: C, 59.72; H, 4.13; N, 17.04 .

\section{Compound $\mathbf{6 d}$}

Crystallized from methanol as white crystals, yield 84\%, mp 120-122 ${ }^{\circ} \mathrm{C} . \mathrm{M} / \mathrm{z}$ (Int.\%): 351 (0.5), 257 (36.36), 201 (45.0), 180 (100), 152 (87.88), 139 (33.33), 124 (39.39), 96 (54.55).

\section{Compound $\boldsymbol{6 e}$}

Crystallized from methanol as white crystals, yield 87\%, mp 248-249 ${ }^{\circ} \mathrm{C} .{ }^{1} \mathrm{H}$ NMR $\left(\delta\right.$, DMSO): 3.77 (s, $3 \mathrm{H}, \mathrm{CH}_{3} \mathrm{O}$ ), 3.84 (s, $\left.6 \mathrm{H}, 2 \mathrm{CH}_{3} \mathrm{O}\right), 6.85$ (s, $2 \mathrm{H}, \mathrm{Ar}-\mathrm{H}$ of trimethoxy ring), 7.34 and 8.16 (each d, $2 \mathrm{H}$ of $\mathrm{CH}=\mathrm{CH}$ ), 7.49-7.78 ppm (m, $4 \mathrm{H}, \mathrm{Ar}-\mathrm{H}), 8.42$ (bs, $1 \mathrm{H}, \mathrm{NH}$ of triazinone). Anal. Calc. for $\mathrm{C}_{26} \mathrm{H}_{18} \mathrm{ClN}_{5} \mathrm{O}_{5}$ (515.92): C, 60.53; H, 3.52; N, 13.57. Found: C, 60.42; H, 3.41; N, 13.44 .

\section{Compound $6 f$}

Crystallized from ethanol as white crystals, yield $85 \%$, $\mathrm{mp}>300^{\circ} \mathrm{C} .{ }^{1} \mathrm{H}$ NMR $(\delta$, DMSO): 7.25 and 8.25 (each d, $2 \mathrm{H}$ of $\mathrm{CH}=\mathrm{CH}$ ), 7.35-7.82 ppm (m, 8H, Ar-H), 8.50 (bs, $1 \mathrm{H}, \mathrm{NH}$ of triazinone). ${ }^{13} \mathrm{C}$ NMR $(\delta$, DMSO $) 75.19\left(\mathrm{C}_{9}-\right.$ $\mathrm{CN}), 87.28\left(\mathrm{C}_{7}-\mathrm{CN}\right), 116.21(\mathrm{C} \equiv \mathrm{N}), 117.09(\mathrm{C} \equiv \mathrm{N}), 122.75$ $\left(\mathrm{C}_{\alpha}\right.$ of $\left.\mathrm{C}=\mathrm{C}\right), 129.50-138.56$ (12C of aryl groups and $\mathrm{C}_{\beta}$ of $\mathrm{C}=\mathrm{C}), 148.46\left(\mathrm{C}_{8}\right), 157.48\left(\mathrm{C}_{9 \mathrm{a}}\right), 157.77\left(\mathrm{C}_{3}\right), 159.26$ $\left(\mathrm{C}_{2}\right.$ as $\left.\mathrm{C}=\mathrm{O}\right)$ and $159.99 \mathrm{ppm}\left(\mathrm{C}_{6}\right.$ as $\left.\mathrm{C}=\mathrm{O}\right)$. Anal. Calc. for $\mathrm{C}_{23} \mathrm{H}_{11} \mathrm{Cl}_{2} \mathrm{~N}_{5} \mathrm{O}_{2}$ (460.28): C, 60.02; H, 2.41; N, 15.22 . Found: C, 60.00; H, 2.34; N, 15.10 . 


\section{Compound $6 \mathrm{~g}$}

Crystallized from methanol as white crystals, yield $65 \%, \mathrm{mp}>300^{\circ} \mathrm{C} . \mathrm{M} / \mathrm{z}$ (Int.\%): 429 (0.19), 285 (100), 256 (25.84), 173 (10.76), 146 (1.17) and 111 (26.39).

8-Aryl-2,3,6-trioxo-1,2,3,4,5,6-hexahydropyrido[1,2-b] [1,2,4]triazine-7,9-dicarbonitriles $(7 \boldsymbol{a}, \boldsymbol{b})$

\section{Method 1}

A mixture of $1 \mathbf{a}$ or $\mathbf{1 b}(0.01 \mathrm{~mol})$ and diethyl oxalate $(0.01 \mathrm{~mol})$ in dry dioxane $(50 \mathrm{~mL})$ was refluxed for $4 \mathrm{~h}$, after cooling the reaction mixture was concentrated. The solid obtained was filtered and crystallized to give $\mathbf{7 a}, \mathbf{b}$.

\section{Method 2}

Compound 1a or $\mathbf{1 b}(10 \mathrm{mmol})$ was dissolved in DMF $(50 \mathrm{~mL})$, oxalyl chloride $(10 \mathrm{mmol})$ was added dropwise within $15 \mathrm{~min}$. The reaction mixture was refluxed for $4 \mathrm{~h}$, after cooling the reaction mixture was poured into ice. The solid obtained was filtered and crystallized to give $\mathbf{7 a}, \mathbf{b}$.

\section{Compound $7 a$}

Crystallized from benzene as white crystals, yield $82 \%$, mp 146-147 ${ }^{\circ} \mathrm{C} . \mathrm{UV} \lambda_{\max } / \mathrm{nm}(\log \varepsilon): 349$ (0.131), 273 (2.857). IR (KBr) $v_{\max } / \mathrm{cm}^{-1}: 3269,3195(2 \mathrm{NH}), 2989,2969,2935$, 2904, $2841\left(\mathrm{CH}_{3}\right.$ groups), $2255(2 \mathrm{C} \equiv \mathrm{N}), 1741$ (weak band for $\mathrm{C}=\mathrm{O}$ ), 1462, 1424 (def. $\mathrm{CH}_{3}$ ). ${ }^{1} \mathrm{H}$ NMR ( $\delta$, DMSO) 3.65 (s, $\left.3 \mathrm{H}, \mathrm{CH}_{3} \mathrm{O}\right)$, and $3.80\left(\mathrm{~s}, 6 \mathrm{H}, 2 \mathrm{CH}_{3} \mathrm{O}\right), 5.10(\mathrm{~s}, 2 \mathrm{H}, 2 \mathrm{OH}$ of 1,2,4-triazinediol), 6.72 (s, $2 \mathrm{H}, \mathrm{Ar}-\mathrm{H}) .{ }^{13} \mathrm{C}$ NMR ( $\delta$, DMSO): $56.73\left(2 \mathrm{CH}_{3} \mathrm{O}\right), 60.84\left(\mathrm{CH}_{3} \mathrm{O}\right), 107.57\left(\mathrm{C}_{7}-\mathrm{CN}\right.$ and $\left.\mathrm{C}_{9}-\mathrm{CN}\right)$, $115.01(2 \mathrm{C} \equiv \mathrm{N}), 131.12$ (aromatic carbons), $137.93\left(\mathrm{C}_{8}\right), 153.70$ ppm ( $3 \mathrm{C}=\mathrm{O}$ and $\mathrm{C}_{9 \mathrm{a}}$ ). Anal. Calc. for $\mathrm{C}_{18} \mathrm{H}_{13} \mathrm{~N}_{5} \mathrm{O}_{6}(395.33)$ : $\mathrm{C}$, 54.69; H, 3.31; N, 17.71. Found: C, 54.67; H, 3.30; N, 17.73.

\section{Compound $7 \boldsymbol{b}$}

Crystallized from DMF as yellow crystals, yield $80 \%, \mathrm{mp}>$ $300{ }^{\circ} \mathrm{C} .{ }^{1} \mathrm{H}$ NMR $(\delta$, DMSO): 7.54 (d, 2H, Ar-H), 7.63 (d, $2 \mathrm{H}, \mathrm{Ar}-\mathrm{H}$ ), $8.39 \mathrm{ppm}$ (s, $2 \mathrm{H}, 2 \mathrm{OH}$ of 1,2,4-triazinediol). ${ }^{13} \mathrm{C}$ NMR ( $\delta$, DMSO): $74.31\left(\mathrm{C}_{9}-\mathrm{CN}\right), 86.54\left(\mathrm{C}_{7}-\mathrm{CN}\right), 115.18$ $(\mathrm{C} \equiv \mathrm{N}), 115.98(\mathrm{C} \equiv \mathrm{N}), 128.69,129.90,133.39$ and 135.07 (6C of aryl carbons), $156.49\left(\mathrm{C}_{8}\right), 158.27\left(\mathrm{C}_{9 \mathrm{a}}\right), 158.90\left(\mathrm{C}_{2}\right.$ and $\mathrm{C}_{3}$ as $\left.2 \mathrm{C}=\mathrm{O}\right), 171.77 \mathrm{ppm}\left(\mathrm{C}_{6}\right.$ as $\left.\mathrm{C}=\mathrm{O}\right) . \mathrm{M} / \mathrm{z}$ (Int.\%): 339 (33.33), 187 (100), 175 (23.81), 142 (54.76), 124 (60.61) and 86 (42.42). Anal. Calc. for $\mathrm{C}_{15} \mathrm{H}_{6} \mathrm{ClN}_{5} \mathrm{O}_{3}$ (339.69): C, 53.04; H, 1.78; N, 20.62. Found: C, 52.91; H, 1.75; N, 20.58.

\section{2,3-Dimethyl-6-oxo-8-(3,4,5-trimethoxy)-6H- pyrido[1,2-b][1,2,4]triazine-7,9-dicarbonitrile (8)}

A mixture of 1a $(5 \mathrm{mmol})$ and butane-2,3-dione $(5 \mathrm{mmol})$ in glacial acetic acid $(20 \mathrm{~mL})$ was refluxed for
$2 \mathrm{~h}$, after cooling the reaction mixture was concentrated. The solid obtained was filtered, washed with cold ethanol and crystallized from acetic acid to give $\mathbf{8}$ as yellow crystals, yield $70 \%, \mathrm{mp}>300{ }^{\circ} \mathrm{C}$. IR (KBr) $v_{\max } / \mathrm{cm}^{-1}: 2926,2849$ $\left(\mathrm{CH}_{3}\right.$ groups), $2213(2 \mathrm{C} \equiv \mathrm{N}), 1696(\mathrm{C}=\mathrm{O}), 1635(\mathrm{C}=\mathrm{N})$, $1585(\mathrm{C}=\mathrm{C}), 1466,1412$ (def. $\left.\mathrm{CH}_{3}\right) .{ }^{1} \mathrm{H}$ NMR $(\delta$, DMSO): $3.20\left(\mathrm{~s}, 3 \mathrm{H}, \mathrm{CH}_{3}\right), 3.62\left(\mathrm{~s}, 3 \mathrm{H}, \mathrm{CH}_{3}\right), 3.75\left(\mathrm{~s}, 3 \mathrm{H}, \mathrm{CH}_{3} \mathrm{O}\right)$, 4.01 (s, $6 \mathrm{H}, 2 \mathrm{CH}_{3} \mathrm{O}$ ), $6.65 \mathrm{ppm}$ (s, $\left.2 \mathrm{H}, \mathrm{Ar}-\mathrm{H}\right) . \mathrm{M} / \mathrm{z}$ (Int.\%): 391 (2.99), 365 (4.25), 337 (6.77), 224 (5.20), 197 (8.50), 171 (21.42), 167 (15.91), 137 (21.57), 109 (38.92), 82 (37.46), 55 (100). Anal. Calc. for $\mathrm{C}_{20} \mathrm{H}_{17} \mathrm{~N}_{5} \mathrm{O}_{4}$ (391.39): C, 61.23; H, 2.55; N, 17.88. Found: C, 60.94; H, 2.10; N, 17.50 .

8-Aryl-2,3-diphenyl-6-oxo-5,6-dihydropyrido[1,2-b] [1,2,4]triazine-7,9-dicarbonitriles $(\mathbf{9 a}, \boldsymbol{b})$

A mixture of $1 \mathbf{a}$ or $\mathbf{1 b}(5 \mathrm{mmol})$ and benzil $(5 \mathrm{mmol})$ in glacial acetic acid $(50 \mathrm{~mL})$ and anhydrous sodium acetate $(1 \mathrm{~g})$ was refluxed for $8 \mathrm{~h}$, after cooling the reaction mixture was poured onto ice. The solid obtained was filtered and crystallized to give 9a,b. For compound 9a; Crystallized from $\mathrm{DMF} / \mathrm{H}_{2} \mathrm{O}$ as yellow crystals, yield $60 \%, \mathrm{mp} 265^{\circ} \mathrm{C}$. UV $\lambda_{\max } / \mathrm{nm}(\log \varepsilon): 347$ (4.33), 282 (4.084). IR (KBr) $v_{\max } / \mathrm{cm}^{-1}: 2935,2861\left(\mathrm{CH}_{3}\right.$ groups $), 2215(2 \mathrm{C} \equiv \mathrm{N}), 1674$ $(\mathrm{C}=\mathrm{O}), 1611(\mathrm{C}=\mathrm{N}), 1593(\mathrm{C}=\mathrm{C}), 1466,1418\left(\right.$ def. $\left.\mathrm{CH}_{3}\right)$. ${ }^{1} \mathrm{H}$ NMR ( $\delta$, DMSO): 3.78 (s, 3H, $\mathrm{CH}_{3} \mathrm{O}$ ), 3.80 (s, $2 \mathrm{H}, 2$ $\mathrm{CH}_{3} \mathrm{O}$ ), 6.97 (s, 2H, Ar-H of trimethoxy ring), 7.14-7.52 ppm (m, 10H, Ar-H). M/z (Int.\%): 515 (45.83), 426 (41.67), 328 (100), 232 (41.67), 221 (45.83) and 147 (45.83). Anal. Calc. for $\mathrm{C}_{30} \mathrm{H}_{21} \mathrm{~N}_{5} \mathrm{O}_{4}(515.53)$ : C, 69.83; H, 4.07; N, 13.58 . Found: C, 70.06; H, 3.97; N 13.54.

\section{Compound $\mathbf{9 b}$}

Crystallized from $\mathrm{DMF} / \mathrm{H}_{2} \mathrm{O}$ as yellow crystals, yield $54 \%, \mathrm{mp}>300^{\circ} \mathrm{C}$. IR $(\mathrm{KBr}) v_{\max } / \mathrm{cm}^{-1}: 2219(2 \mathrm{C} \equiv \mathrm{N}), 1669$ $(\mathrm{C}=\mathrm{O}), 1608(\mathrm{C}=\mathrm{N}), 1585(\mathrm{C}=\mathrm{C}) .{ }^{1} \mathrm{H}$ NMR $(\delta, \mathrm{DMSO})$ : 7.19-7.55 ppm (m, 10H, Ar-H), 7.72 (d, 2H, Ar-H), 7.83 (d, $2 \mathrm{H}, \mathrm{Ar}-\mathrm{H})$. Anal. Calc. for $\mathrm{C}_{27} \mathrm{H}_{14} \mathrm{ClN}_{5} \mathrm{O}$ (459.89): C, 70.52; H, 3.07; N, 15.23. Found: C, 70.36; H, 3.02; N, 15.20.

\section{8-Aryl-2,3-diphenyl-6-oxo-1,2,5,6-tetrahydropyrido[1,2-b]} [1,2,4]triazine-7,9-dicarbonitriles $(\mathbf{1 0 a}, \boldsymbol{b})$

\section{Method 1}

A mixture of 1a or $1 \mathbf{b}(5 \mathrm{mmol})$ and benzoin $(5 \mathrm{mmol})$ in glacial acetic acid $(50 \mathrm{~mL})$ and anhydrous sodium acetate $(1 \mathrm{~g})$ was refluxed for $8 \mathrm{~h}$, after cooling the reaction mixture was poured onto ice. The solid obtained was filtered, washed several times with water and crystallized to give 10a,b. 


\section{Method 2}

Compounds $\mathbf{1 0 a}$ or $\mathbf{1 0 b}(5 \mathrm{mmol})$ was dissolved in methanol $(50 \mathrm{~mL})$, ferric chloride $(10 \%, 20 \mathrm{~mL})$ in methanol $(30 \mathrm{~mL})$ was added and refluxed for $3 \mathrm{~h}$, after cooling the reaction mixture was concentrated. The solid obtained was filtered and crystallized to give 9a,b. Melting point and mixed melting point showed no depression with 9a,b obtained from the above experiment.

\section{Compound 10a}

Crystallized from $\mathrm{DMF} / \mathrm{H}_{2} \mathrm{O}$ as yellow crystals, yield $49 \%$, mp 278-279 ${ }^{\circ} \mathrm{C}$. IR (KBr) $v_{\max } / \mathrm{cm}^{-1}: 3449(\mathrm{NH})$, 2977, 2944, $2846\left(\mathrm{CH}_{3}\right), 2212(2 \mathrm{C} \equiv \mathrm{N}), 1650(\mathrm{C}=\mathrm{O}), 1591$ $(\mathrm{C}=\mathrm{N}), 1562(\mathrm{C}=\mathrm{C}), 1458,1416\left(\right.$ def. $\left.\mathrm{CH}_{3}\right)$. Anal. Calc. for $\mathrm{C}_{30} \mathrm{H}_{23} \mathrm{~N}_{5} \mathrm{O}_{4}$ (517.55): C, 69.62; H, 4.48; N, 13.53. Found: C, 69.50; H, 4.35; N 13.38.

\section{Compound $\mathbf{1 0 b}$}

Crystallized from $\mathrm{DMF} / \mathrm{H}_{2} \mathrm{O}$ as white crystals, yield 44\%, mp 290-291 ${ }^{\circ} \mathrm{C} .{ }^{1} \mathrm{H}$ NMR ( $\delta$, DMSO): 5.62 (s, 1H, $\mathrm{CH}$ of 1,2,4-triazin-5-yl), 7.21-8.05 (m, 14H, Ar-H), 8.53 ppm (s, 1H, NH). ${ }^{13} \mathrm{C}$ NMR ( $\delta$, DMSO): $75.19\left(\mathrm{C}_{9}-\mathrm{CN}\right)$, $77.83\left(\mathrm{C}_{2}\right), 87\left(\mathrm{C}_{7}-\mathrm{CN}\right), 117.09(2 \mathrm{C} \equiv \mathrm{N}), 129.51-135.95$ (18C of aryl carbons), $157.49\left(\mathrm{C}_{8}\right), 159.27\left(\mathrm{C}_{9 \mathrm{a}}\right), 170.78$ $\left(\mathrm{C}_{3}\right), 194.49$ ppm $\left(\mathrm{C}_{6}\right.$ as $\left.\mathrm{C}=\mathrm{O}\right)$. Anal. Calc. for $\mathrm{C}_{27} \mathrm{H}_{16} \mathrm{ClN}_{5} \mathrm{O}$ (461.91): C, 70.21; H, 3.49; N, 15.16. Found: C, 70.09; H, 3.42; N, 15.08 .

\section{2,3-Dichloro-6-oxo-8-(3,4,5-trimethoxy)-6H-pyrido[1,2-b] [1,2,4]triazine-7,9-dicarbonitrile (11)}

A mixture of 7a $(5 \mathrm{mmol})$ and phosphorus oxychloride $(10 \mathrm{~mL})$ was refluxed for $2 \mathrm{~h}$, after cooling the reaction mixture was poured onto ice with stirring. The solid obtained was filtered, washed several times with water and crystallized from benzene to give $\mathbf{1 1}$ as white crystals, yield $55 \%, \mathrm{mp}>300^{\circ} \mathrm{C}$. IR (KBr) $v_{\max } / \mathrm{cm}^{-1}: 2964,2936,2842$ $\left(\mathrm{CH}_{3}\right.$ groups), $2240(2 \mathrm{C} \equiv \mathrm{N}), 1680(\mathrm{C}=\mathrm{O}), 1605(\mathrm{C}=\mathrm{N})$, $1582(\mathrm{C}=\mathrm{C}), 1473,1414\left(\right.$ def. $\left.\mathrm{CH}_{3}\right) .{ }^{1} \mathrm{H}$ NMR $(\delta, \mathrm{DMSO})$ : $3.79\left(\mathrm{~s}, 3 \mathrm{H}, \mathrm{CH}_{3} \mathrm{O}\right), 3.83\left(\mathrm{~s}, 6 \mathrm{H}, 2 \mathrm{CH}_{3} \mathrm{O}\right)$ and $6.81(\mathrm{~s}, 2 \mathrm{H}$, Ar-H). Anal. Calc. for $\mathrm{C}_{18} \mathrm{H}_{11} \mathrm{Cl}_{2} \mathrm{~N}_{5} \mathrm{O}_{4}$ (432.22): C, 50.20; H, 2.57; N, 16.20. Found: C, 50.31; H, 2.46; N, 16.24.

8-Aryl-2,3,6-trioxo-1,4-dihydroxymethyl-1,2,3,4,5,6hexahydropyrido[1,2-b][1,2,4]triazine-7,9-dicarbonitriles $(12 a, b)$

A mixture of $7 \mathbf{a}$ or $\mathbf{7 b}(5 \mathrm{mmol})$ and formaldehyde solution $(10 \mathrm{mmol})$ in methanol $(50 \mathrm{~mL})$ was refluxed for $6 \mathrm{~h}$. The solid obtained after cooling was filtered, washed several times with water and crystallized to give 12a,b.

\section{Compound $12 a$}

Crystallized from methanol as yellow crystals, yield $73 \%$, mp 214-215 ${ }^{\circ} \mathrm{C}$. UV $\lambda_{\text {max }} / \mathrm{nm}(\log \varepsilon): 348$ (1.89), 337 (1.54), 269 (2.55). IR (KBr) $v_{\max } / \mathrm{cm}^{-1}: 3399,3280(2$ $\mathrm{OH}), 2940,2940,2839\left(\mathrm{CH}_{3}\right), 2215(2 \mathrm{C} \equiv \mathrm{N}), 1660(\mathrm{C}=\mathrm{O})$, $1595(\mathrm{C}=\mathrm{C}), 1467,1414$ (def. $\left.\mathrm{CH}_{3}\right) .{ }^{1} \mathrm{H}$ NMR $(\delta, \mathrm{DMSO})$ : 3.25 (s, 4H, 2- $\left.\mathrm{CH}_{2}-\right), 3.75$ (s, 3H, $\left.\mathrm{CH}_{3} \mathrm{O}\right), 3.81$ (s, 6H, 2 $\left.\mathrm{CH}_{3} \mathrm{O}\right), 5.68$ (s, 1H, OH), 6.84 (s, 1H, Ar-H), 6.86 (s, 1H, Ar-H), 8.41 ppm (s, $1 \mathrm{H}, \mathrm{OH})$. Anal. Calc. for $\mathrm{C}_{20} \mathrm{H}_{17} \mathrm{~N}_{5} \mathrm{O}_{8}$ (455.39): C, 52.70; H, 3.37; N, 15.37. Found: C, 52.52; $\mathrm{H}, 3.15 ; \mathrm{N}, 15.25$.

\section{Compound $\mathbf{1 2 b}$}

Crystallized from methanol as yellow crystals, yield $69 \%, \mathrm{mp} 219-220{ }^{\circ} \mathrm{C}$. IR (KBr) $v_{\max } / \mathrm{cm}^{-1}: 3293,3212$ (2 OH), 2990, $2932\left(\mathrm{CH}_{2}\right), 2217(2 \mathrm{C} \equiv \mathrm{N}), 1667(\mathrm{C}=\mathrm{O})$, 1618 (def. $\mathrm{OH}$ ), 1497, 1450 (def. $\mathrm{CH}_{2}$ ). M/z (Int.\%) 399 (26.19), 239 (35.17), 187 (100), 142 (26.19), 124 (28.57), 112 (30.95) and 88 (26.19).

9-Aryl-5,11-dihydroxymethyl-7-oxo-2-thioxo-2,3,5,6,7,11hexahydropyrido[1,2:2',3'] triazino[5',6'-f]triazine-8,10dicarbonitriles $(\mathbf{1 3 a}, \boldsymbol{b})$

A mixture of $\mathbf{1 2 a}$ or $\mathbf{1 2 b}(2 \mathrm{mmol})$ and thiosemicarbazide (2 mmol dissolved in hot water) in acetic acid $(40 \mathrm{~mL})$ was refluxed for $4 \mathrm{~h}$. The solid obtained after cooling was filtered and crystallized to give $13 \mathbf{a}, \mathbf{b}$.

\section{Compound $13 a$}

Crystallized from acetic acid as yellow crystals, yield $77 \%, \mathrm{mp}>300{ }^{\circ} \mathrm{C}$. UV $\lambda_{\max } / \mathrm{nm}(\log \varepsilon): 379$ (3.9), 345 (4.4). IR (KBr) $v_{\max } / \mathrm{cm}^{-1}: 3412,3281(2-\mathrm{OH}), 3214(\mathrm{NH})$, 2973, 2941, $2840\left(\mathrm{CH}_{3}\right), 2213(2 \mathrm{C} \equiv \mathrm{N}), 1670(\mathrm{C}=\mathrm{O})$, $1609(\mathrm{C}=\mathrm{N}), 1513(\mathrm{C}=\mathrm{C}), 1468,1413\left(\right.$ def. $\left.\mathrm{CH}_{3}\right), 1184$ $(\mathrm{C}=\mathrm{S}) .{ }^{1} \mathrm{H}$ NMR $(\delta, \mathrm{DMSO}): 3.04$ (s, 4H, $\left.2 \mathrm{CH}_{2} \mathrm{O}-\right), 3.77$ (s, 3H, $\left.\mathrm{CH}_{3} \mathrm{O}\right), 3.80$ (s, 6H, $\left.2 \mathrm{CH}_{3} \mathrm{O}\right), 5.95$ (s, $\left.1 \mathrm{H}, \mathrm{OH}\right)$, 6.84 (s, 1H, Ar-H), 6.89 (s, 1H, Ar-H), 8.63 (s, 1H, OH), 10.25 ppm (s, 1H, NH). Anal. Calc. for $\mathrm{C}_{21} \mathrm{H}_{18} \mathrm{~N}_{8} \mathrm{O}_{6} \mathrm{~S}$ (510.49): C, 49.41; H, 3.55; N, 21.94. Found: C, 49.94; H, 4.10; N, 22.10 .

\section{Compound $\mathbf{1 3 b}$}

Crystallized from acetic acid as yellow crystals, yield $65 \%, \mathrm{mp}>300{ }^{\circ} \mathrm{C} . \lambda_{\max } / \mathrm{nm}(\varepsilon): 346$ (3.4), 273 (2.98). IR $(\mathrm{KBr}) v_{\max } / \mathrm{cm}^{-1}: 3416(\mathrm{OH}), 3344(\mathrm{OH}), 3307(\mathrm{NH})$, $2217(\mathrm{C} \equiv \mathrm{N}), 1668(\mathrm{C}=\mathrm{O}), 1613(\mathrm{C}=\mathrm{N}), 1549(\mathrm{C}=\mathrm{C})$. ${ }^{1} \mathrm{H}$ NMR ( $\delta$, DMSO): 2.87 (s, 4H, $2 \mathrm{CH}_{2}$ ), 5.43 (s, 2H, $2 \mathrm{OH}), 7.54$ (d, 2H, Ar-H), 7.65 (d, 2H, Ar-H), 9.89 ppm (s, 1H, 1NH). 
9-(4-Chlorophenyl)-5,11-dihydroxymethyl-7-oxo-2hydrazino-2, 3,5, 6,7,11-hexahydropyrido [1,2:2',3'] triazino[5',6'-f]triazine-8,10-dicarbonitrile (14)

A mixture of $\mathbf{1 3 b}(10 \mathrm{mmol})$ and hydrazine hydrate $(100 \mathrm{mmol})$ in absolute ethanol $(100 \mathrm{~mL})$ was refluxed for $12 \mathrm{~h}$. The solid obtained after cooling was filtered and crystallized from DMF to give $\mathbf{1 4}$ as yellow crystals, yield $54 \%, \mathrm{mp}>300^{\circ} \mathrm{C}$. IR (KBr) $v_{\max } / \mathrm{cm}^{-1}: 3470(\mathrm{OH}), 3304$, $3187\left(\mathrm{NH}, \mathrm{NH}_{2}\right), 2926\left(\mathrm{CH}_{2}\right), 2214(2 \mathrm{C} \equiv \mathrm{N}), 1636(\mathrm{C}=\mathrm{O})$, $1579(\mathrm{C}=\mathrm{N}), 1489$ (def. $\left.\mathrm{CH}_{2}\right) .{ }^{1} \mathrm{H}$ NMR $(\delta$, DMSO): 2.73 (s, $2 \mathrm{H}, \mathrm{CH}_{2}$ ), 2.89 (s, $2 \mathrm{H}, \mathrm{CH}_{2}$ ), 5.09 (bs, $\left.2 \mathrm{H}, \mathrm{NH}_{2}\right), 5.75$ (s, 2H, 2OH), 7.54 (d, 2H, Ar-H), 7.65 (d, 2H, Ar-H), 7.94 ppm (s, 1H, 1NH). Anal. Calc. for $\mathrm{C}_{18} \mathrm{H}_{13} \mathrm{ClN}_{10} \mathrm{O}_{3}(452.82)$ : C, 47.70; H, 2.87; N, 30.92. Found: C, 47.84; H, 2.53; N, 30.89 .

6-Amino-4-(3,4,5-trimethoxyphenyl)-2-oxo-1-[2-oxo-1,2dihydro-3-indolo-3-ylidine)amino]-1,2-dihydropyridine3,5-dicarbonitrile (15)

A mixture of $1 \mathbf{a}(5 \mathrm{mmol})$ and isatine $(5 \mathrm{mmol})$ in ethanol $(75 \mathrm{~mL})$ and piperidine (2 drops) was refluxed for $4 \mathrm{~h}$. The solid obtained was filtered and crystallized from ethanol to give $\mathbf{1 5}$ as yellow crystals, yield 51\%, $\mathrm{mp}>300{ }^{\circ} \mathrm{C}$. IR (KBr) $v_{\max } / \mathrm{cm}^{-1}: 3310,3172\left(\mathrm{NH}_{2}\right.$, $\mathrm{NH}), 2921,2851\left(\mathrm{CH}_{3}\right), 2207(2 \mathrm{C} \equiv \mathrm{N}), 1646(\mathrm{C}=\mathrm{O})$, $1518(\mathrm{C}=\mathrm{N}), 1496,1442\left(\right.$ def. $\left.\mathrm{CH}_{3}\right) .{ }^{1} \mathrm{H}$ NMR $(\delta$, DMSO): 3.03 (s, $1 \mathrm{H}, \mathrm{NH}), 3.79$ (s, $\left.3 \mathrm{H}, \mathrm{CH}_{3} \mathrm{O}\right), 3.83$ (s, $\left.3 \mathrm{H}, \mathrm{CH}_{3} \mathrm{O}\right), 3.85\left(\mathrm{~s}, 3 \mathrm{H}, \mathrm{CH}_{3} \mathrm{O}\right), 6.97(\mathrm{~s}, 2 \mathrm{H}, \mathrm{Ar}-\mathrm{H}$ of trimethoxy phenyl), 7.39-7.99 (m, 4H, Ar-H of indole), $8.28 \mathrm{ppm}\left(\mathrm{s}, 2 \mathrm{H}, \mathrm{NH}_{2}\right)$. Anal. Calc. for $\mathrm{C}_{24} \mathrm{H}_{18} \mathrm{~N}_{6} \mathrm{O}_{5}$ (470.45): C, 61.28; H, 3.86; N, 17.86. Found: C, 61.19; $\mathrm{H}, 3.84 ; \mathrm{N}, 17.81$.

3-(2-Aminophenyl)-8-(4-chlorophenyl)-2,6-dioxo1,6-dihydro-2H-pyrido[1,2-b][1,2,4]triazine-7,9dicarbonitrile (16)

A mixture of 1a $(5 \mathrm{mmol})$ and isatine $(5 \mathrm{mmol})$ in alcoholic $\mathrm{NaOH}(5 \%, 50 \mathrm{~mL})$ was refluxed for $4 \mathrm{~h}$, cooled and neutralized with conc. HCl. The solid so formed was filtered, washed several times with water and crystallized from $\mathrm{DMF} / \mathrm{H}_{2} \mathrm{O}$ to give $\mathbf{1 6}$ as yellow crystals, yield $46 \%$, $\mathrm{mp}>300{ }^{\circ} \mathrm{C}$. IR $(\mathrm{KBr}) v_{\max } / \mathrm{cm}^{-1}: 3500-3336(\mathrm{~b}, \mathrm{OH}$, $\left.\mathrm{NH}_{2}\right), 2200(2 \mathrm{C} \equiv \mathrm{N}), 1621(\mathrm{C}=\mathrm{O}), 1548(\mathrm{C}=\mathrm{N}) .{ }^{1} \mathrm{H}$ NMR ( $\delta$, DMSO): 4.87 (bs, $2 \mathrm{H}, \mathrm{NH}_{2}$ ), 7.23-8.10 (m, 8H, Ar-H), $11.62 \mathrm{ppm}$ (bs, $1 \mathrm{H}, \mathrm{NH}$ ). Anal. Calc. for $\mathrm{C}_{21} \mathrm{H}_{11} \mathrm{ClN}_{6} \mathrm{O}_{2}$ (414.81): C, 60.81; H, 2.67; N, 20.26. Found: C, 61.04; H, 2.61; N, 20.14.
8-Aryl-10-oxo-11-hydroindolo[2,3-e]pyrido[1,2-b][1,2,4] triazine-7,9-dicarbonitriles $(\mathbf{1 7 a} \boldsymbol{a} \boldsymbol{b})$

Compound 15 or $16(2 \mathrm{mmol})$, glacial acetic acid $(50 \mathrm{~mL})$ and anhydrous sodium acetate $(1 \mathrm{~g})$ was refluxed for $12 \mathrm{~h}$, after cooling the reaction mixture was concentrated. The solid so formed was filtered and crystallized to give 17a,b (Table 2).

\section{Compound 17a}

Crystallized from acetic acid as orange crystals, yield $73 \%, \mathrm{mp}>300^{\circ} \mathrm{C}$. IR $(\mathrm{KBr}) \mathrm{v}_{\max } / \mathrm{cm}^{-1}: 3491(\mathrm{NH})$, 2996, 2943, $2834\left(\mathrm{CH}_{3}\right), 2218(2 \mathrm{C} \equiv \mathrm{N}), 1667(\mathrm{C}=\mathrm{O})$, $1640(\mathrm{C}=\mathrm{N}), 1587(\mathrm{C}=\mathrm{C}), 1474,1428\left(\right.$ def. $\left.\mathrm{CH}_{3}\right) . \mathrm{M} / \mathrm{z}$ (Int.\%): 452 (3.27), 377 (4.24), 337 (6.63), 339 (12.68), 309 (6.34), 172 (23.44), 145 (40.44), 117 (65.03), 108 (36.31), 90 (19.88). Anal. Calc. for $\mathrm{C}_{24} \mathrm{H}_{16} \mathrm{~N}_{6} \mathrm{O}_{4}(452.43)$ : C, 63.71; H, 3.56; N, 18.58. Found: C, 63.59; H, 3.49; N, 18.45 .

\section{Compound $\mathbf{1 7 b}$}

Crystallized from DMF as orange-yellow crystals, yield $84 \%, \mathrm{mp}>300^{\circ} \mathrm{C}$. IR $(\mathrm{KBr}) v_{\max } / \mathrm{cm}^{-1}: 3381(\mathrm{NH})$, $2202(2 \mathrm{C} \equiv \mathrm{N}), 1622(\mathrm{C}=\mathrm{O}) .{ }^{1} \mathrm{H}$ NMR $(\delta$, DMSO): 6.80$7.82(\mathrm{~m}, 8 \mathrm{H}, \mathrm{Ar}-\mathrm{H}), 12.30 \mathrm{ppm}(\mathrm{s}, 1 \mathrm{H}, \mathrm{NH}) .{ }^{13} \mathrm{C}$ NMR $(\delta$, DMSO): $75.19\left(\mathrm{C}_{7}-\mathrm{CN}\right), 87.29\left(\mathrm{C}_{9}-\mathrm{CN}\right), 116.22(\mathrm{C} \equiv \mathrm{N})$, $117.09(\mathrm{C} \equiv \mathrm{N}), 129.65-135.95$ (18C of aryl carbons), $157.48\left(\mathrm{C}_{8}\right.$ and $\left.\mathrm{C}_{12 \mathrm{a}}\right), 159.26\left(\mathrm{C}_{5 \mathrm{a}}\right), 160\left(\mathrm{C}_{6 \mathrm{a}}\right), 163.17$ $\mathrm{ppm}\left(\mathrm{C}_{10}\right.$ as $\left.\mathrm{C}=\mathrm{O}\right) . \mathrm{M} / \mathrm{z}$ (Int.\%): 396 (3.39), 368 (9.05), 319 (15.49), 285 (12.14), 254 (41.10), 209 (6.03), 117 (13.50), 111 (64.20), 57 (100). Anal. Calc. for $\mathrm{C}_{21} \mathrm{H}_{9} \mathrm{ClN}_{6} \mathrm{O}$ (396.80): C, 63.57; H, 2.29; N, 21.18. Found: C, 63.42; H, 2.25; N, 20.98.

8-(3,4,5-Trimethoxy)-5-acetyl-10-oxo- 11 hydroindolo[2,3-e]pyrido[1,2-b][1,2,4]triazine-7,9dicarbonitrile (18)

A mixture of $\mathbf{1 7 a}(5 \mathrm{mmol})$ and acetic anhydride $(10 \mathrm{~mL})$ was refluxed for $2 \mathrm{~h}$. The solid obtained after cooling was filtered, washed with cold ethanol and crystallized from acetic acid to give $\mathbf{1 8}$ as yellow crystals, yield $80 \%, \mathrm{mp}>300^{\circ} \mathrm{C}$. IR (KBr) $v_{\max } / \mathrm{cm}^{-1}: 2999,2947$, $2840\left(\mathrm{CH}_{3}\right), 2216(2 \mathrm{C} \equiv \mathrm{N}), 1695(\mathrm{C}=\mathrm{O}), 1604(\mathrm{C}=\mathrm{N})$, $1583(\mathrm{C}=\mathrm{C}), 1477,1416$ (def. $\left.\mathrm{CH}_{3}\right) . \mathrm{M} / \mathrm{z}$ (Int.\%): 494 (5.57), 452 (3.27), 337 (4.62), 327 (5.89), 311 (7.01), 299 (6.69), 211 (8.76), 185 (5.57), 133 (8.28), 116 (10.35), 93 (10.67) and 55 (100). Anal. Calc. for $\mathrm{C}_{26} \mathrm{H}_{20} \mathrm{~N}_{6} \mathrm{O}_{5}$ (494.47): C, 63.16; H, 3.67; N, 17.00. Found: C, 63.10; $\mathrm{H}, 3.54$; N, 16.84 . 
8-(3,4,5-Trimethoxyphenyl)-2-(2-acetylaminophenyl)3,6-dioxo-3,6-dihydro-4H-pyrido[1,2-b] triazine-7,9dicarbonitrile (20)

A mixture of 1a $(5 \mathrm{mmol})$ and $\mathrm{N}$-acetylisatine $(5 \mathrm{mmol})$ in absolute ethanol $(100 \mathrm{~mL})$ and few drops of piperidine was refluxed for $4 \mathrm{~h}$, after cooling the reaction mixture was concentrated. The solid obtained was filtered, washed with cold ethanol and crystallized from DMF to give $\mathbf{2 0}$ as orange crystals, yield $65 \%, \mathrm{mp}>300{ }^{\circ} \mathrm{C}$. IR $(\mathrm{KBr})$ $v_{\max } / \mathrm{cm}^{-1}: 3452,3283(2 \mathrm{NH}), 2939,2838\left(\mathrm{CH}_{3}\right), 2213$ $(2 \mathrm{C} \equiv \mathrm{N}), 1673,1680(3 \mathrm{C}=\mathrm{O}), 1618(\mathrm{C}=\mathrm{N}), 1589(\mathrm{C}=\mathrm{C})$, 1490, 1415 (def. $\left.\mathrm{CH}_{3}\right) .{ }^{1} \mathrm{H}$ NMR $(\delta, \mathrm{DMSO}): 2.02(\mathrm{~s}, 3 \mathrm{H}$, $\left.\mathrm{COCH}_{3}\right), 3.79\left(\mathrm{~s}, 3 \mathrm{H}, \mathrm{CH}_{3} \mathrm{O}\right), 3.83\left(\mathrm{~s}, 6 \mathrm{H}, 2 \mathrm{CH}_{3} \mathrm{O}\right), 5.69$ (s, 1H, NH), 6.85 (s, 1H, Ar-H of trimethoxy phenyl), 6.96 (s, 1H, Ar-H trimethoxy phenyl), 7.27 (s, 1H, Ar-H), 7.57 (s, 1H, Ar-H), 7.94 ppm (s, 2H, Ar-H), 10.8 (s, 1H, NH). Anal. Calc. for $\mathrm{C}_{26} \mathrm{H}_{20} \mathrm{~N}_{6} \mathrm{O}_{6}(512.48): \mathrm{C}, 60.94 ; \mathrm{H}, 3.93 ; \mathrm{N}$, 16.40. Found: C, 60.91; H, 3.88; N, 16.35 .

\section{References}

1. Abdel-Monem, W. R.; Chem. Pap. 2004, 58, 276; Attaby, F. A.; Eldin, S. M.; Abdel- Razik, M.; Phosphorus, Sulfur Silicon Relat. Elem. 1995, 21, 106; Elnagdi, M. H.; Ghozlan, S. A.; Abdel-Razik, F. M.; Maghraby, A. S.; Chem. Synop. 1991, 5, 116.

2. Fronabarger, J. W.; Chapman, R. D.; Gilardi, R. D.; Tetrahedron Lett. 2006, 47, 7707; Bhosale, R. S.; Sarda, S. R.; Ardhapure, S. S.; Jadhav, W. N.; Bhusare, S. R.; Pawar, R. P.; Tetrahedron Lett. 2005, 46, 7183; Harb, A. A.; Chem. Pap. 2004, 58, 260; Kuethe, J. T.; Wong, A.; Davies, I. W.; J. Org. Chem. 2004, 69, 7752.

3. Ganjali, M. R.; Norouzi, P.; Faridbod, F.; Ghorbani, M.; Adib, M.; Anal. Chim. Acta 2006, 569, 35; Gustaffon, B.; Hakansson, M.; Hutton, A. T.; Moss, J. R.; Jagner, S.; Inorg. Chim. Acta 2005, 358, 1327; Na, H.; Lee, D. C.; Lim, J. W.; Choi J. H.; Byun, J. C.;
Park, Y. C.; Polyhedron 2002, 21, 917; Boghaei, D. M.; Mohebi, S.; Tetrahedron 2002, 58, 5357; Jeewoth, T.; Bhowon, M. G.; Wah, H. L. K.; Transition Met. Chem. 1999, 24, 445.

4. Abdel-Rahman, R. M.; Trends Heterocycl. Chem. (India) 2002, 8, 187; Abdel- Rahman, R. M.; Pharmazie 2001, 56, 275; AbdelRahman, R. M.; Pharmazie 2001, 56, 195.

5. Al-Najjar, A. A. A.; Amer, S. A.; Riad, M.; Elghamy, I.; Elnagdi, M. H.; J. Chem. Res. 1996, 296.

6. Gindets, V. P.; Kherson, I.; Povstyanoi, M. V.; Kapov, P. B.; Grandberg, I. I.; Ukr. Khim. Zh. 1988, 54, 531.(CA 110:75450v); Kang-Chien, L. S.; Jane-Bi; Tao-Tung, M.; Zhonghua Yaoxue Zazhi 1993, 45, 89. (CA 119:203378c); Yamada, Y.; Kurokawa, S.; Yasuda, H.; Utsunomiya Daigaku Kyoikugakubkiyo 1993, 43, 35. (CA 120:19162z).

7. El-Gendy, Z.; Morsy, J. M.; Allimony, H. A.; Abdel-Monem, W. R.; Abdel-Rahman, R. M.; Phosphorus, sulfur silicon Relat. Elem. 2003, 178, 2055.

8. Abdel-Rahman, R. M.; Morsy, J. M.; Allimony, H. A.; AbdelMonem, W. R.; Boll. Chin. Farmaceutico 1999, 138, 176.

9. Seada, M.; Abdel-Rahman, R. M.; Hanafy, F.; Indian J. Chem. Soc. 1992, 69, 882.

10. Juhasz-Riedel, Z.; Hajious, G.; Kollenz, R.; Massmer, A.; Chem. Ber. 1989, 122, 1935. (CA 112:7454r); Riedel, Z.; Hajious, G.; Kollenz, R.; Massmer, A.; Monatsh Chem. 1992, 123, 1181. (CA 118:254860u).

11. Wiedermannova, I.; Jirovsky, D.; Hlavac, J.; Slouka, J.; Chemica 2000, 39, 69; Wiedermannova, I.; Slouka, J.; Hlavac, J.; Chemica 1999, 38, 83.

12. Dandi, A.; Sanno, S.; Bhagat, S.; Arya, K.; Six International Electronic Conference on Synthetic Organic Chemistry, ecsoc-6, 2002; http://www. mdpi.net/ecsoc-6.

13. Gould, J. C.; Bowie, J. M.; Edinb. Med. J. 1952, 59, 198; Singh, A.; Latita, R.; Dhakarey, R.; Saxena, G.; J. Indian Chem. Soc. 1996, 73, 339 .

Received: September 16, 2008 Web Release Date: June 4, 2009 